\title{
Human Hair Perforation: An Additional Tool in Forensic Biology
}

\section{Itisha Singh*}

Department of Microbiology, Saaii College of Medical Sciences and Technology, Kanpur, India

\begin{abstract}
The hair perforation is a prominent feature in several infected hairs. Different types of perforators are found in human hairs. Some of these are presented in the present study for the first time which may be proved as additional tool in hair diagnosis in forensic biology. Other structure and type of hairs, their chemical structures are also reviewed in order to consolidate important aspects of hair diagnosis.
\end{abstract}

Keywords: Human hair; Perforation; Tool; Forensic biology

\section{Introduction}

Presence of hair on skin is unique feature of human beings. Each hair consist of the part within the follicles the hair root and shaft projecting the body surface. The hair is nourished by a hair follicle. Hair follicle produced keratinized cell which push the shaft from below and spread the oily secretion over the shaft. Due to such type of secretion hair is protected against adverse environment. Entire hair consist of three regions medulla in the middle, a thicker cortex around medulla and a delicate covering of cuticle which is made up of keratinous cells. Cortex formed by fusi form fibres which contain granules of melanin but medulla are without pigment. Keratin is protective layer of hair; it differs from other protein on the basis of high sulphur containing amino acids, cystine, cysteine and methionine. The sulpur is found mainly in the disulphide bonds of cystine molecules which cross link adjacent peptide chains. The disulphide bonds are considered to be responsible for the stability of keratin. Hair are differentiated with characteristic length, color, shape, root appearance, and internal microscopic. Considerable variability also exists in the types of hairs that are found on the body. In humans, hairs found on the head, pubic region, arms, legs, and other body areas have characteristics that can determine their origin. Because hairs can be transferred during physical contact, their presence can associate a suspect to a victim or a suspect to a crime scene. The types of hair recovered and the condition and number of hairs found all impact on their value as evidence in a criminal investigation. Comparison of the microscopic characteristics of questioned hairs to known hair samples helps determine whether a transfer may have occurred.

Hair evidence is very important because of the transference or exchange phenomenon. Hair samples are often critical clues to identifying the perpetrator of a broad range of crimes, including rape, burglary, assault, and vehicular homicide. The phenotypic characteristics of human hairs are assessed by observing the microscopic anatomy of a hair and estimating the body area, racial. The examination of human hairs in the forensic laboratory is typically conducted through the use of light microscopy. The purpose for conducting this examination is to ascertain whether two or more individuals could have come into contact or whether one or more individuals could have come into contact with an object. This associative evidence is particularly useful in crimes of violence, such as homicide, sexual assault, and aggravated assault, where physical contact may have occurred. Crimes such as burglary and armed robbery typically involve the recovery of debris and articles of clothing which may contain hairs useful for the identification of suspects.

\section{Hair structure and growth}

Hair is present on many different regions of the body. Each region, such as the head, pubic area, chest, axillae, and limbs, has hairs with microscopical characteristics attributable to that region. Although it is possible to identify a hair as originating from a particular body area, the regions of the body that are primarily used in forensic comparisons are the head and pubic areas. As hairs undergo a cyclical growth (anagen) and resting phase (telogen), the visible microscopic characteristics are sufficient to determine the phase of growth of the hair. During the anagen phase, the hair is actively growing, and materials are deposited in the hair shaft by cells found in the follicle. Metabolically active and dividing cells above and around the dermal papilla of the follicle grow upward during this phase, to form the major components of the hairthe medulla, cortex, cuticle, and accompanying root sheath. In the telogen phase, the follicle is dormant or resting. The transition period between the anagen and telogen phases is referred to as the catagen phase.

\section{Human hairs}

Physical contact may result in the transfer of hairs. These can transfer directly from the region of the body where they are growing or they can transfer from the clothing of individuals. These hairs are shed on clothing and on items in the environment. Contact between a victim and a suspect's environment can easily cause a secondary transfer of hair. Hairs that are found on the clothing of suspects or victims and appear to have fallen out naturally may be the result of transfer. Hairs that have been forcibly removed may suggest a violent confrontation.

\section{Materials and Methods}

Hair taken from human beings is examined under Olympus BX40 Trinocular Microscope after mounting them in PVA cotton blue fluid. Different types of perforator observed were photographed using Olympus digital camera. Other features were reviewed from literature received from various sources.

*Corresponding author: Itisha Singh, Department of Microbiology, Saai College of Medical Sciences and Technology, Kanpur, India, Tel: 07376139540; E-mail: itisha.micro@gmail.com

Received Maerch 20, 2014; Accepted October 20, 2014; Published October 25, 2014

Citation: Singh I (2014) Human Hair Perforation: An Additional Tool in Forensic Biology. J Forensic Res 5: 244 doi:10.4172/2157-7145.1000244

Copyright: (c) 2014 Singh I. This is an open-access article distributed under the terms of the Creative Commons Attribution License, which permits unrestricted use, distribution, and reproduction in any medium, provided the original author and source are credited. 


\section{Hair perforation}

The hair perforation is a prominent feature in several infected hairs. Different types of perforators are found in human hairs. Some of these are presented in this study for the first time which may be proved as additional tool in hair diagnosis in forensic biology [Figures 1-3]. Detailed human hair perforating study has already been made by Kushwaha et al. [1], Bahuguna and Kushwaha [2,3].

\section{Determination of hair}

The analyst may use chemical, macroscopic, or microscopic techniques to make that finding. The chemical technique is a test of the hair's ability to burn. Vegetable fibers burn rather easily, emit a smell like burnt wood, and leave a sharply burnt end. Additionally, burning hair creates a smell like burning feathers, and burnt hair usually has a fused or rounded shaft end. Hairs ordinarily grow singly

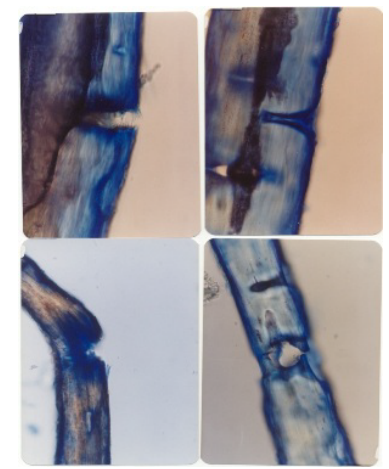

Figure 1: Different types of hair perforators leading to hair damage $X 100$.

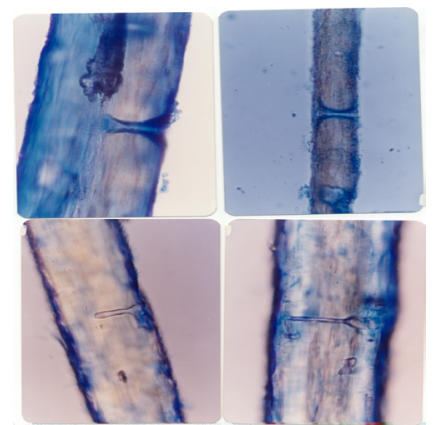

Figure 2: Long perforators in human hair X100.

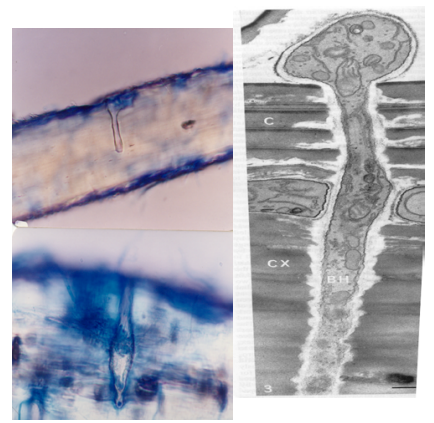

Figure 3: SEM of hair perforator [4]. without branching. Vegetable fibers are readily distinguishable because they often grow in tight bunches and exhibit branching. The analyst can detect these characteristics easily. The microscopic technique is probably the most trustworthy method of determining whether the sample is hair. In length wise view of a hair strand, the one can observe a tip end, a shaft, and a bulb or root end. In cross-sectional view, there are three concentric rings. The core is the medulla, containing cellular debris and pigment granules of melanin. The middle ring is the cortex, which also includes pigment. The cortex is composed of flat, elongated cells. The outermost layer is the surface cuticle, which consists of overlapping scales. The scales are transparent and point toward the tip end of the hair strand.

\section{Difference between human and animal hair}

There are other features such as cuticle scale width that are indicative of human origin, but the most important assessment is that the metrical features of human head hair are not distinct enough from those of other primates to use micro-measurement as a sole means of hair identification method. In addition to the micro-measurement technique, there are macroscopic and microscopic methods of differentiating human and animal hair. The macroscopic technique involves examining the hair's gross characteristics. Animal hair varies in type; many animals have both heavy guard hair and finer fur hair. Human hair is unitary in type.

Human hair tends to have a narrow central modularly canal. The human medulla is normally fragmented and has no obvious cell pattern. The human medulla approximates one third of the width of the cortex. Animal hair differs in each respect. The hair of lower animals have a broad medulla, display a clear cellular structure, and usually have medullas more than half as broad as the cortical shell. The human cortex layer contains the majority of the pigment granules while, in other mammals, most pigment is located in the medulla. In the human cortex, the pigment granules are packed most densely toward the peripheral areas. There is often a central accumulation of pigment cells in animal hair. The cuticle or scale shell is also additional feature in distinguishing between human and animal hair. The scales of human hair overlap smoothly, but those of other mammalian species often protrude roughly. None of these characteristics is exclusively human, but the cumulative presence of these characteristics makes it almost certain that the hair is human in origin.

\section{Unaltered hair and weathering of hair}

Unaltered human hair is hair that has not been chemically modified by treatment with bleaches, permanent waves, strengtheners, or hair dyes. Weathering effects in human hair may be explored by comparing tip ends (longer exposed) to root ends [5].

\section{Bleached and permanent-waved hair}

The whole-fiber amino acid composition of human hair bleached on the head with commercial hair-bleaching agents-alkaline hydrogen peroxide or alkaline peroxide/persulfate has been described in the literature [6,7]. Nineteen amino acids in human hair have been studied for possible modification during permanent waving.

\section{Aging effect on hair}

As a person ages, hormonal changes contribute to changes in the hair, in addition to hair thickness, hair graying, hair diameter and dryness of the scalp and the hair. Hair thinning tends to relate to hair density and therefore to not to be noticeable in women until the mid to late twenties or more commonly a few years later. Diameter tends to 
peak at about age 20 for men and in the 40 for women.

\section{The effects of menopause on the hair}

A decrease in softness and smoothness of the hair of postmenopausal women has also been reported. It is also likely that hair curvature increases with advancing age as has been shown for women. This effect will likely make the hair fibers more prone to frizziness and will decrease hair luster. The effects of these age related changes in hair density, diameter or area of cross-section, graying, curvature and hair lipids produce changes in fiber properties which produce changes in important consumer hair assembly properties such as a changes in combing ease, hair body, hair coverage, frizziness, manageability, style retention, etc. Aging of individual hair fibers on one's head by everyday grooming actions generally results in a gradual degradation of the scales through cuticle fragmentation. This process is related to the actual age or time the individual hair has been on the scalp rather than to the chronological age of the individual.

\section{The variation of hair diameter with age and sex}

Graying of hair relates to the size and distribution of the melanin granules as well as the types of pigments in the fibers. Gray hair is also dependent on the production of less pigment in the individual hairs with advancing age. Children's hair is finer, thinner, and less welldeveloped. However, the state of the art does not enable the analyst to be more specific. The other method is chemical test. The test involves dissolving the hair in a solution of caustic potash. The faster the roots dissolve, the younger the human hair source. The speed of dissolution is relative and varies, and for that reason the caustic potash test does not permit an exact age estimate.

\section{Lipids in hair and trace metals in hair}

The total amount of lipid extractable from hair is generally 1-9\% of the weight of the hair [8]. Masukawa et al. [8] studied the total hair lipid composition. There are a number of studies describing the quantitative determination of various elements of human hair other than carbon, hydrogen, nitrogen, oxygen, and sulfur. In particular, the inorganic constituents of human hair appear to be receiving some attention because of their potential in diagnostic medicine and to a lesser degree in forensic science. The mineral content of human hair fibers is generally very low (less than $1 \%$ ).

\section{Heavy metals in hair}

If a person was killed by arsenic poisoning, there will be an arsenic residue in the hair. Proof of the arsenic residue can be important evidence of the homicidal character of the death. Although heavy metals occur at low concentrations in human hair, they sometimes accumulate at concentrations well above those levels present in blood or urine. Concentrations of metals such as cadmium, arsenic, mercury, and lead in hair tend to correlate with the amounts of these same metals in internal organs [8]. Furthermore, it is well known that human hair serves as a tissue for the localization of arsenic during arsenic poisoning.

\section{Male or female human hair}

There are a number of techniques for determining the sex of the person who was the source of the human hair strand. The oldest method relies upon the strands' gross characteristics. Male hair is generally larger in diameter, shorter in length, and wirier in texture. The average diameter of male human hair is $1 / 350$ inch while that of female hair is approximately $1 / 450 \mathrm{inch}$. Until the last decade, women routinely manipulated their hair more extensively than men by pinning, curling, brushing, and combing. The manipulation frequently caused the tip ends of female hair to split. Men have begun to treat their hair more frequently, and consequently the presence of a split end now has much less evidentiary value. One technique is the test for the $\mathrm{X}$-chromatin body in the hair root sheath. Roughly sixty percent of the cells of a normal woman will include the chromatin body. The presence of X-chromatin bodies is a specific test, since the frequencies do not overlap. There are differences in trace element concentrations between male and female hair. This test is not as conclusive as the X-chromatin or Y-chromosome test, but it is a helpful, confirmatory test. One cannot determine race as positively as sex, but the analyst nevertheless can make a relatively confident finding as to the race of the human hair source. The identification of race is most useful as an investigative tool, but it can also be an associative tool when an individual's hairs exhibit unusual racial characteristics.

\section{Region of the body area determination}

Microscopy also can enable the analyst to determine the part of the human body that a hair strand came from. Scalp or crown hair tends to be soft and long. Scalp hair ordinarily displays less diameter variation and more even pigment distribution than hair from other regions of the body. Eyebrow, eyelash, and nostril hairs are short, stiff, and thick. They have wide medullas, and they taper very quickly to a fine point. Beard and moustache hairs are the thickest. Beard hair is much curved and coarse. These hairs are irregular in shape and have a triangular cross-section. Hairs from the chest or back seem immature. They are generally fine, short, and flexible. Hairs from legs and arms are short, fine, and contain little pigment. Pubic and auxiliary hairs are wiry. Because there is a wide range of interpersonal variation in head and pubic hairs, the majority of work in forensics has been in comparing and differentiating hairs from the head and pubic regions. Pubic hairs are generally coarse and wiry in appearance. They exhibit considerable diameter variation or buckling and often have a continuous to discontinuous medulla. While tapered tips are common, these hairs may also be abraded or cut.

\section{Determining whether the hair is natural or dyed, fell out or pulled, cut or crushed}

Natural hair can be distinguished from dyed hair microscopically. Dyed hair has a duller appearance, and the inner margin of its cuticle is obscured. If hair falls out naturally, the strand will have its root end. The presence of a root is powerful evidence that the hair fell out due to natural causes such as disease. Such a hair will have a clean appearance. The appearance of a pulled hair differs markedly. The pulled strand will seem mutilated, and the bulb may have a portion of the sheath clinging to it. If the hair was cut, the cortical shells of the shaft will display a clean, smooth slice. However, if the assailant used a blunt instrument, the cortical cells will have a jagged, crushed, or rough appearance.

\section{Blood group of the hair}

During the past decade, the general field of serology or blood analysis progressed as rapidly as any scientific discipline [9]. The same holds true for the specific application of serology to hair analysis. In certain circumstances, the analyst can determine the $\mathrm{ABO}$ blood grouping of the human hair source.

\section{Acknowledgement}

I am thankful to DST for financial assistance and The Chairman and Director of Saaii College of Medical Sciences, Kanpur for providing research facilities. I am also grateful to Dr. J.P. Mall, MS, Director, Mall Hospital and Research Centre, Lucknow and Dr. N.P. Mall, MBBS for constant encouragement. 


\section{References}

1. Bahuguna S, Kushwaha RK (1989) Hair perforation by keratinophilic fungi. Mycoses 32: 340-343.

2. Bahuguna S, Kushwaha RK (1993) Influence of different oils on penetration of human hair by fungi. Int J Cosmet Sci 15: 1-5.

3. Kushwaha RKS, Sarika Katiyar, Divya Bhadauria (2002) In: Keratinophilic fungi and hair invasion. Recent Advances in Forensic Biology. Guru AK, Shrivastava P, Sagar SFL (eds.) Anmol Publications, Delhi. 79-89.

4. Kushwaha RKS, Guarro J (2000) Biology of keratinophilic fungi. Rev. Iberoamericana Micologia, Bilbao, Spain.
5. Veldsman DP (1966) Weathering in wool. Part 3: the chemical effects of weathering. Wool Sci Rev 29: 33-44

6. Robbins CR, Kelly CH (1969) Amino acid analysis of cosmetically altered hair J Soc Cosmet Chem 20: 555-564

7. Furman OS, Teel AL, Watts RJ (2010) Mechanism of base activation of persulfate. Environ Sci Technol 44: 6423-6428.

8. Masukawa Y, Narita H, Imokawa G (2005) Characterization of the lipid composition at the proximal root regions of human hair. J Cosmet Sci 56: 1-16.

9. Maugh TH $2^{\text {nd }}$ (1978) Hair: a diagnostic tool to complement blood serum and urine. Science 202: 1271-1273 